\title{
Single-Center Experience in Targeted Prostate Biopsy Using Multiparametric Magnetic Resonance Imaging-Transrectal Ultrasound Elastic Fusion Technique
}

\begin{abstract}
Introduction: Transrectal ultrasound (TRUS)-guided random biopsies are used to be the gold standard when diagnosing prostate cancer. A relatively new system with organ tracking that fuses real-time TRUS images with previously acquired multiparametric magnetic resonance imaging (mpMRI) images for prostate biopsy guidance is presented here. The primary goal of the study is to correlate (1) the mpMRI findings with the Gleason score grading of the prostate biopsies performed under mpMRI-TRUS elastic fusion and (2) the prostate-specific antigen (PSA) levels with the Gleason grading. Materials and Methods: Between January 2017 and August 2018, 58 patients had targeted prostate biopsy using mpMRI-TRUS elastic fusion technique (Urostation). These patients had previously the mpMRI of the prostate at our center using three-dimensional T2-weighted imaging, diffusion-weighted imaging, and dynamic contrast enhanced imaging. Of all 58 patients, 32 patients were classified as having Prostate Imaging-Reporting And Data System (PI-RADS) 4/5, 21 patients as PI-RADS 3, and five as PI-RADS 2. Results: Twenty-seven patients had positive biopsies for prostate cancer. Positive results were found in 25 patients having PI-RADS $4 / 5$ (25 out of 32). Of these patients, 20 had positive specimens from the dominant lesion, four from both the targeted lesion and nontargeted areas, and one from a nontargeted area. Positive results were found in two patients classified as PI-RADS 3 from targeted and nontargeted areas. These results show that $78 \%$ of the patients classified by mpMRI as PI-RADS $4 / 5$ and $10 \%$ of the patients classified as PI-RADS 3 had positive biopsies for prostate cancer. The results also showed a correlation between the PI-RADS score on mpMRI, the Gleason score, and the PSA levels. Conclusion: mpMRI-TRUS fusion biopsy is a safe and accurate method for targeted prostate biopsies. Our preliminary results are comparable to the published international numbers and show a good correlation between the PI-RADS classification and histopathology, as well as correlation between PI-RADS, Gleason scores, and PSA levels of positive biopsies.
\end{abstract}

Keywords: Gleason score, multiparametric magnetic resonance imaging, multiparametric magnetic resonance imaging/transrectal ultrasound elastic fusion biopsy, Prostate Imaging-Reporting And Data System, prostate-specific antigen, prostate, targeted biopsy

\section{Introduction}

Prostate cancer is the second most common cancer in men. In fact, about one in seven men will be diagnosed with this disease during their lifetime. However, most cases are detected at an early stage and are low risk, posing little or no threat to the patient. Biopsy is required for diagnosis and grading. The gold standard method still present in the guidelines uses a conventional two-dimensional (2D) transrectal ultrasound (TRUS) guidance for prostate biopsy. ${ }^{[1,2]}$ This method is not however standardized, as significant difference

This is an open access journal, and articles are distributed under the terms of the Creative Commons Attribution-NonCommercial-ShareAlike 4.0 License, which allows others to remix, tweak, and build upon the work non-commercially, as long as appropriate credit is given and the new creations are licensed under the identical terms.

For reprints contact: WKHLRPMedknow_reprints@wolterskluwer.com in prostate cancer detection rates exists among operators. It has poor detection rates not exceeding $40 \%$ at the first biopsy run and $20 \%$ at the second biopsy run, due to ineffective sampling. ${ }^{[3]}$ It can be harmful to the patients when considering that $1 \%$ will develop prostatitis, which may lead to urosepsis in a third of these cases, and one-third of those developing urosepsis will die. ${ }^{[4-6]}$

The TRUS/multiparametric magnetic resonance imaging (mpMRI) fusion biopsy was thus introduced as a safe and accurate method and revolutionized the concept of the prostate biopsy from a

How to cite this article: Farah J, El-Hajj G, Assaf E, Noufaily A, Chamsuddin A, Jabbour M, et al. Singlecenter experience in targeted prostate biopsy using multiparametric magnetic resonance imagingtransrectal ultrasound elastic fusion technique. Arab J Intervent Radiol 2020;4:111-6.

\section{Jennifer Farah, Gerard EI-Hajj, Edward Assaf', Abdallah Noufaily, Abbas Chamsuddin, Michel Jabbour ${ }^{1}$, Fatmeh Ghandour'2, Emilie Fayad, Raja Ashou}

Departments of Radiology, ${ }^{1}$ Urology and ${ }^{2}$ Anatomic Pathology, Saint George University Medical Center, University of Balamand, Achrafieh, Beirut, Lebanon

Received: 25-11-2019 Revised: 22-01-2020 Accepted: 06-02-2020 Online Published: 25-04-2020

Address for correspondence: Dr. Jennifer Farah, Department of Radiology, Saint George University Medical Center, University of Balamand, P.O. Box 166378, Achrafieh, Beirut 1100 2807, Lebanon. E-mail: jennifernfarah@gmail. com

\section{Access this article online}

Website: www.arabjir.com

DOI: 10.4103/AJIR.AJIR_32_19 Quick Response Code:

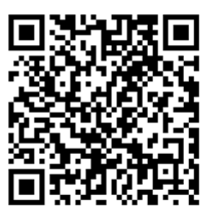


totally nontargeted approach to a more precise and targeted technique having less harmful complications for the patient. Using the transperineal (TP) approach will avoid the risk of urosepsis while the transrectal approach will decrease the number of biopsies by targeting the dominant lesion. ${ }^{[7]}$

\section{Background}

Nontargeted prostate biopsies done under TRUS guidance are prone to sampling error. ${ }^{[8]}$ They have high rates of false-positive results diagnosing clinically nonsignificant cancer and high rates of false-negative results missing clinically significant cancer. $^{[8]}$ mpMRI brought better resolution, better anatomical visualization, and most importantly the capability to visualize clinically significant prostate cancer, as well as functional assessment. It also allows assessment of tumor aggressiveness. ${ }^{[8]}$ It combines high-resolution T2-weighted image (T2WI) with functional techniques such as diffusion-weighted imaging and dynamic contrast-enhanced, allowing better delineation of the anatomy, better evaluation of both peripheral and transition zones, as well as better evaluation of the tumor vascularity. It adds to the sensitivity and specificity of diagnosing prostate cancer. ${ }^{[8]}$

Targeted biopsy techniques have then emerged. These included the in-bore magnetic resonance (MR)-guided fusion which can be cumbersome, the cognitive MR-ultrasound (MR-US) fusion, and the device-mediated MR-US fusion. ${ }^{[8]}$ Biopsies targeted using MR images combined with real-time 3D TRUS allow us to reliably identify the spatial location of the index lesion in the prostate and characterize its aggressiveness.

The mostly recognized indications of the mpMRI/TRUS fusion biopsy include biopsy-naïve men, men with prior negative biopsy, men with low-risk disease considering active surveillance, men with enlarged prostate $>40 \mathrm{~mL}$, and men with guided registration for focal therapy. ${ }^{[9]}$

Trinity offers multimodality images with the possibility of fusing, with high accuracy, the images of mpMRI as well as positron emission tomography (PET)-computed tomography with the dedicated Koelis console for performing and recording targeted and nontargeted biopsies, both by the transrectal and TP approaches. [10-12] The biopsies' information is digitized and can be recalled for quality control, rebiopsy, or planning targeted treatment. ${ }^{[13]}$

In comparing among the mostly recognized devicemediated fusion platforms (UroNav, Artemis and Urostation), Urostation Trinity (Koelis) offers unique features of an US image acquisition. It relies on automatic instead of manual probe rotation, elastic image instead of rigid image registration and fusion. It also uses image-based (TRUS-TRUS) registration tracking mechanism, taking into account patient and prostate movement and deformation instead of electromagnetic tracking or mechanical arm with encoders. Urostation has a 3-s delay for image acquisition. ${ }^{[9]}$
Previous cognitive and device-mediated techniques were totally based on the US probe position, did not account for the movement of the patient and the prostate as well as prostate deformation, were real time, but had a rigid fusion mechanism. ${ }^{[9]}$ In a prospective study comparing 12-core random systematic biopsy and 4-core targeted biopsy, cognitive and device-mediated fusion techniques had similar results. Similar results were also reported in the PROFUS trial (prospective blinded comparison). ${ }^{[8]}$ In a study of 391 patients, device-mediated fusion was better at detecting clinically significant cancer and limiting clinically nonsignificant cancer detection. ${ }^{[8]}$ The PROMIS study also showed comparable results, with an $18 \%$ increase in significant cancer detection, 5\% decrease in nonsignificant cancer detection, thus avoiding biopsy in $27 \%$ of patients. ${ }^{[14]}$ The PRECISION study also revealed the increased yield of detecting less nonsignificant cancer, avoiding biopsies using targeted versus standard biopsy approach. ${ }^{[3]}$ Other studies showed a higher detection rate of significant cancer with four instead of two targeted cores. ${ }^{[15]}$ Device-mediated fusion also has a role in ruling out clinically significant cancer, with studies showing that none of the men with negative MRI had any Gleason more than $4 .^{[16]}$ The men with Prostate Imaging-Reporting And Data System (PI-RADS) 1-2 had 100\% Negative predictive value (NPV) for the detection of Gleason 4/5. ${ }^{[17]}$ In men with prostate-specific antigen (PSA) $<10 \mathrm{mg} / \mathrm{dL}$, Prostate Volume (PV) $<33 \mathrm{~mL}$, normal MRI was associated with NPV of $93.7 \%-97.5 \%$ for clinically significant disease. ${ }^{[18]}$ Other studies showed that MRI-TRUS fusion biopsies increased the yield of first-round prostate biopsies in patients with a prostate volume $>40 \mathrm{~mL}$. ${ }^{[19]}$

The mpMRI/TRUS fusion technique has a role in ruling in clinically significant cancer. Diagnosis of high-risk disease was made $30 \%$ more than with the standard biopsy while diagnosis of low-risk disease was made $<17 \%$ with the standard biopsy. ${ }^{[20]}$ It showed increased cancer length per biopsy core and better detection of high-grade cancer with high Gleason score and of clinically significant cancer, with improved clinical-pathological correlation. ${ }^{[21]}$ These results were seen in biopsy-naïve men and in men with prior negative biopsy. It has thus a role in reducing over-detection and over-treatment. ${ }^{[9]}$ In men with low-risk cancer, repeat by targeted biopsy improves risk stratification and allows selection of men appropriate for active surveillance without repeating the biopsy. ${ }^{[9]}$

\section{Materials and Methods}

Between January 26, 2017, and August 28, 2018, 58 patients had targeted prostate biopsy using mpMRI-TRUS elastic fusion technique under Trinity. These patients have previously had mpMRI of the prostate at our center or other centers with re-reads and re-classification of PI-RADS from our center using the PI-RADS v2 in our mpMRI reading. The MRI protocol used in our institution (on the 1.5T GE 
Healthcare Signa MRI scanner) included a sagittal, coronal, axial T2weighted FSE (T2wFSE) sequences centered on the prostate (field-of-view [FOV] $21.0 \mathrm{~cm}$, slice thickness $3.5 \mathrm{~mm}$, slice gap $0.3 \mathrm{~mm}$ ) as well as diffusion weighted (DW) sequence (b value of 1000, FOV $33.0 \mathrm{~cm}$, slice thickness $3.5 \mathrm{~mm}$, slice gap $0.3 \mathrm{~mm}$ ), a coronal T2wFSE for the whole pelvis as well as axial T1 LAVA pre and postgadolinium dynamic acquisition for the prostate (FOV $30.0 \mathrm{~cm}$, slice thickness $4 \mathrm{~mm}$, temporal resolution of 10 seconds/acquisition, Matrix 256/256).

All our patients had a fleet enema before the examination. Patients' selection to perform mpMRI was decided by the referring urologists. All the mpMRI prostate readings and reclassification were done by two trained and certified radiologists having attended each at least one workshop in mpMRI of the prostate.

The age range was between 51 and 79 years. Of all 58 patients, 32 patients were classified as having PI-RADS 4/5, 21 patients as PI-RADS 3, and 5 patients as PI-RADS 2. To note that some patients had more than one lesion. In our study, we grouped our patients by PI-RADS category according to the highest PI-RADS of the index lesion. The biopsies of the other lesions were considered as targeted biopsies in the pathology vials and the results of the study, but not in the PI-RADS category. All patients had a recent negative urine culture, underwent bowel preparation, and received antibiotic coverage. Anticoagulation and antiplatelet therapy when applicable was withheld, before the biopsy.

The mpMRI examinations were downloaded by a radiologist, and the 3D-reconstructed images, as well as the target lesions, were placed on the station screen before the biopsy session. About $8 \mathrm{~min}$ was needed to perform this preliminary step. On the examination day, 3D TRUS acquisition was also performed by the radiologist, and the images were fused with the mpMRI-reconstructed images. Ten minutes is also needed to achieve this step.

The biopsy session was performed in the presence of the radiologist and referring urologist, lasting for about $10 \mathrm{~min}$. Nursing staff was always present during all the examination sessions. Ten milliliters of $1 \%$ lidocaine was injected as a local anesthetic at the neurovascular bundle through the transrectal probe before the biopsy. Patients had targeted biopsies of the index lesion and the other lesions if present, and most of them had guided nontargeted registered biopsies in the rest of the prostate zones [Figure 1]. A mean of 6.4 biopsies from the dominant lesions and a mean of five biopsies from the nontargeted areas from the peripheral zones of both lobes were performed. The navigation system used was Urostation Trinity. It uses an elastic image fusion and an organ-based tracking technique, enabling freehand 3D transrectal biopsy mapping and accurate tracking of the prostate, compensating for both patient- and probe-induced mobility. The whole procedure length from image

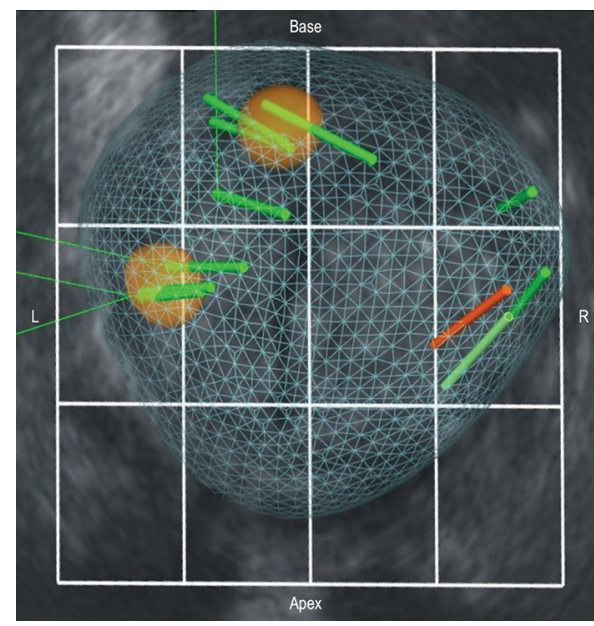

Figure 1: Targeted biopsies from two suspicious nodules in the left transitional zone at the level of the midgland and base, as well as nontargeted biopsies from the right peripheral zone, were taken under Trinity

downloading and fusion to biopsy performance lasted about 30 min. A single radiologist (operator) performed all the examination steps, including the biopsies, whether by himself or by assisting the referring urologists who wanted to acquire the biopsies specimens themselves. The targeted specimens were sent in one vial to the pathology laboratories. Nontargeted specimens were sent in two separate vials and labeled "right" and "left" according to the biopsy side. No reported complications of uncontrolled bleeding or sepsis were noted in all biopsied patients.

\section{Results}

The total number of cores taken was 662. This averages to 11.4 biopsy cores per patient. All five PI-RADS 2 patients had negative results, i.e., nonclinically significant prostate cancer (NCSPC). From the 21 PI-RADS 3 patients, two patients had clinically significant prostate cancer (CSPC) (Gleason 6 and Gleason $7[3+4]$ ), one in the targeted area and the other in the nontargeted area. The other 19 patients had NCSPC.

From the PI-RADS 4 and 5 patients, 25 patients had positive biopsies, 24 were in the targeted areas, and five were in the nontargeted areas. These overlapping results in the targeted and nontargeted areas are because some patients had positive biopsies from both areas [Figure 2]. In particular, two patients with PI-RADS 4 as well as two others with PI-RADS 5 categories were found to have positive biopsies from both areas. This sums up to $78 \%$ positive biopsies in the PI-RADS $4 / 5$ category, $10 \%$ positive biopsies in the PI-RADS 3 category, and $100 \%$ negative biopsies in the PI-RADS 2 category. In total, we had 27 patients with positive biopsies [Table 1].

Nontargeted biopsies were positive in six out of the 27 patients or $22 \%$ of the time. Of these, two patients had only the nontargeted biopsies positive. The CSPC detected 


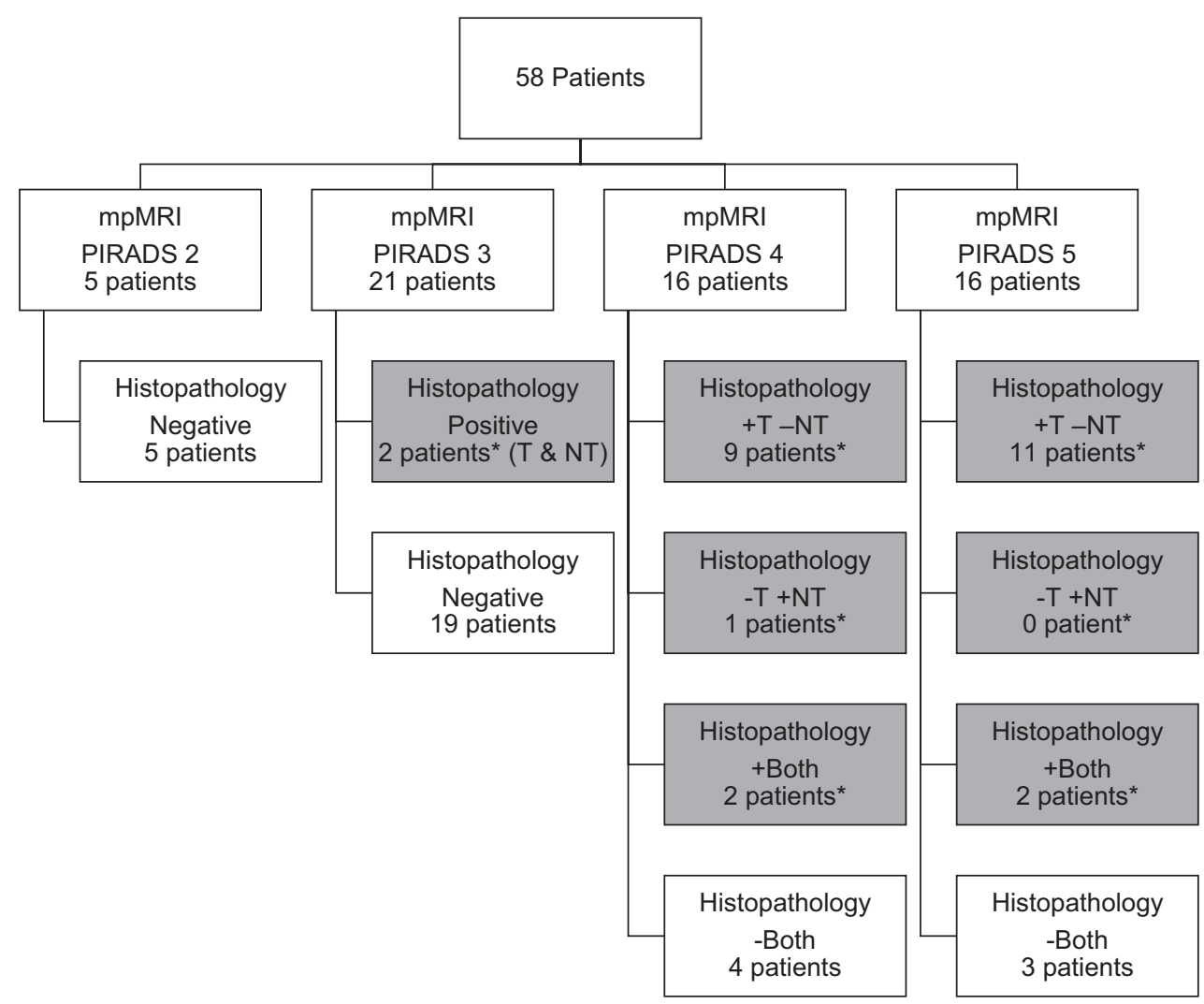

Figure 2: Histopathology results according to PI-RADS classification. Legend: (T: Targeted; NT: Non-targeted) *Clinically significant cancer (CSC): PIRADS 3: 2 patients, PIRADS 4: 12 patients, PIRADS 5: 13 patients. Grey = positive

in the nontargeted areas were primarily adjacent to the targeted areas. Results were also classified according to the Gleason score on the pathology report [Table 1] and according to the PSA levels [Table 2].

All patients with Gleason 8 and 9 are PI-RADS 5. Gleason $7(3+4)$ patients are $92 \%$ PI-RADS $4 / 5$ (mainly 5) and $8 \%$ PI-RADS 3. Gleason 6 patients are 91\% PI-RADS $4 / 5$ (mainly 4 ) and $9 \%$ PI-RADS 3. $90 \%$ of the patients with negative biopsies have PSA $<10.10 \%$ of the patients with negative biopsies have PSA 10-20. Patients with PSA $>20$ have Gleason 7 and 9 only. Only one patient with PSA $<10$ has Gleason 9. Only one patient with PSA 10-20 has Gleason 8.

\section{Discussion}

Many papers have shown that mpMRI/TRUS fusion biopsy of the prostate resulted in increased detection of clinically significant cancer, decreased detection of low-risk cancer, and improvement in patient outcome. ${ }^{[1]}$ Our results show a positive correlation between mpMRI findings and pathology results comparable to the previously published series for patients having biopsies performed under Trinity in 15 European centers. Our study sample was however much smaller as we had difficulty to introduce this new modality in our medical environment. We had to convince the urologists to shift their practice from performing the

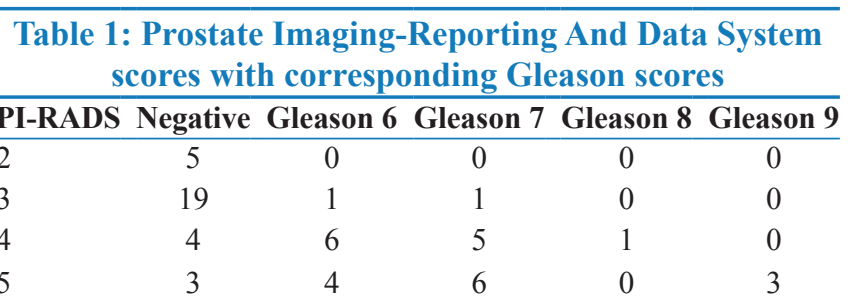

PI-RADS: Prostate Imaging-Reporting And Data System

Table 2: Gleason scores according to prostate-specific antigen levels

\begin{tabular}{lccccc}
\hline $\begin{array}{l}\text { PSA } \\
(\mathbf{n g} / \mathbf{m L} \mathbf{)}\end{array}$ & Negative & Gleason 6 & Gleason 7 & Gleason 8 & Gleason 9 \\
\hline$<10$ & 28 & 10 & 9 & 0 & 1 \\
$10-20$ & 3 & 1 & 1 & 1 & 0 \\
$>20$ & 0 & 0 & 2 & 0 & 2 \\
\hline
\end{tabular}

PSA: Prostate-specific antigen

TRUS non-targeted biopsies under regular US, to the MRIUS fusion technique in the radiology department. Both the specialized radiologist and referring urologist were present during the biopsy session. Only about one-third of the yearly hospital load was transferred to us during the study period.

The mean detection rate for CSPC for PI-RADS 5 lesions was $80 \%$ while for PI-RADS 4 lesions was $50 \%$. In our 
series, it was $75 \%$ for PI-RADS $4,81.25 \%$ for PI-RADS 5, and $78 \%$ for PI-RADS 4 and 5 combined. As for PI-RADS 3 lesions, the CSPC detection rate was around $18 \%$ in the European series, while it was $10 \%$ in our series. ${ }^{[22]} \mathrm{A}$ major limitation of our study was the number of patients enrolled, which was much smaller than the other European studies. We had no positive biopsies in cases labeled PI-RADS 2. We had also a positive correlation between mpMRI PI-RADS and Gleason scoring. All Gleason 8 and above were PI-RADS 4/5. All PI-RADS 3 lesions were Gleason 7 or 6. Most Gleason 7 patients are PI-RADS 4/5.

Nontargeted biopsies were positive in $22 \%$ of the patients. Of them, two patients were positive for nontargeted biopsies only. These results align with the recommendations of few papers in performing targeted and nontargeted biopsies. ${ }^{[23]}$ A good correlation was noted among patients with PSA $<10 \mathrm{ng} / \mathrm{mL}$ having negative biopsies and with patients with PSA $>20 \mathrm{ng} / \mathrm{mL}$ having high Gleason score of more than 7. The presence of negative biopsies for PI-RADS 4/5 lesions may be attributed to the imaging limitations, including sensitivity and specificity of mpMRI, or to inadequate sampling due to the lesion location in the apex and anterior region, and to the fact that all patients with negative biopsies had PSA $<10 \mathrm{ng} / \mathrm{mL}$.

The operator's performance is also crucial. All the required steps are essential to get positive biopsies including proper mpMRI reading, downloading images to Trinity, adequate target placement on the 3D-reconstructed prostate volume, fusion with US images, and adequate biopsy sampling. Inaccurate performance of the operator in any of these steps may result in biopsy failure.

Limitation of positive specimens detected by the TRUS-MRI fusion technique in the apical and anterior lesions was also described by Hossack et al. ${ }^{[2]}$ This highlights the role of performing TP- targeted MRI/US elastic fusion which is a newer technique, also available on Trinity. The TP approach has the advantages of lower risk of infective complications, fever, sepsis, and rectal bleeding. It provides an easier approach to the lesions located in the anterior and apical zones. It is a safe and accurate procedure. However, it requires inpatient stay and deep sedation in comparison to the local anesthesia in TR biopsies. New trends of performing TP biopsy in an outpatient setting under local anesthesia using Trinity have been described and is available online. ${ }^{[13]}$ Both TR and TP biopsies did not show, however, difference in terms of postbiopsy acute retention or hematuria. ${ }^{[2,25]}$

The prostate volume of the patients in our study ranged between 12 and $155 \mathrm{~mL}$, some of them having had prior TURP. However, the prostate volume was not taken into consideration when evaluating the results. The concept of taking the PSA density (PSA-D) in addition to the PI-RADS classification into consideration in deciding for the prostate biopsy performance was not a standard approach when we started our study. de Gorski et al. concluded that TR MRI/US fusion biopsies increased the yield of first-round prostate biopsies in patients with a prostate volume $>40 \mathrm{~cm}^{3} .^{[26]}$ The Cambridge pathway ${ }^{[27]}$ of assessing prostate cancer in 2018 includes TP fusion biopsy for anterior lesions, TR fusion biopsy for posterior lesions, and systemic biopsy for PSA-D $>0.2$ and negative MRI (PI-RADS 2). The PSA-D is calculated by dividing the PSA by the prostate volume. The NPV of MRI increases from 0.84 to 0.91 if the PSA-D $\leq 0.15$ becomes $\leq 0.1$. Our present personal approach in patients selection criteria for prostate biopsies was modified since March 2019. It takes into account the PSA-D in addition to the PIRADS score. We nowadays perform fusion biopsy for patients with PI-RADS 2 if the PSA-D $>0.2$, as well as targeted and nontargeted biopsies for PI-RADS 3 if the PSA-D $>0.12$, and for all the PI-RADS $4 / 5$ patients. We did not also include the target lesion volume in our study, although some articles provide this information.

Another limitation of our study was the nonseparation of the nontargeted lesions in a separate vial. Only two additional vials from the right or left gland zones were sent for all the specimens because of economic issues with the pathology department. As a result, the active surveillance of these lesions would not be precise as for the targeted lesion, if a second-look biopsy is to be taken from the registered biopsy tracts under Trinity. We took too many cores with an average of 11.4 cores per patient, 6.4 from the targeted dominant lesion, and five from nontargeted areas from both peripheral zones. We could have taken less cores from the targeted lesions as described by Baco et al. ${ }^{[7]}$ and hence benefiting from the decreased rate of complications, resulting from the decreased passes numbers. As we are becoming more trained and experienced with the modality, we intend to decrease the number of targeted biopsies from the dominant lesion to 4 instead of 6.4 .

\section{Conclusion}

Trinity provides basic functions for accurate guidance in transrectal and TP MRI/US elastic fusion for prostate cancer, with the availability of a second-look recall capability for future control and monitoring of detected lesions under active surveillance. It can also fuse PET images to evaluate for recurrence and to guide focal therapy after the installation of the complementary microwave Katty system for focal prostate cancer ablation on the Trinity platform. The preliminary clinical trial results of the FOSTINE study at Hospital Cochin, Paris, in focal prostate cancer treatment are very promising in this respect in terms of cancer control and significant decrease in the complications related to prostatic surgery (hematuria, acute urinary symptoms, hematospermia, and rectorrhagia). We hope to be able in the near future to present a new series of patients undergoing focal therapy under Trinity. 
Financial support and sponsorship

Nil.

\section{Conflicts of interest}

There are no conflicts of interest.

\section{References}

1. Peltier A, Aoun F, Lemort M, Kwizera F, Paesmans M, Van Velthoven R. MRI-targeted biopsies versus systematic transrectal ultrasound guided biopsies for the diagnosis of localized prostate cancer in biopsy naïve men. Biomed Res Int. 2015;2015:571708. doi: $10.1155 / 2015 / 571708$.

2. Expert Panel on Urologic Imaging, Coakley FV, Oto A, Alexander LF, Allen BC, Davis BJ, et al. ACR appropriateness criteria ${ }^{\circledR}$ prostate cancer-pretreatment detection, surveillance, and staging. J Am Coll Radiol 2017;14:S245-57.

3. Kasivisvanathan V, Rannikko AS, Borghi M, Panebianco V, Mynderse LA, Vaarala MH, et al. MRI-targeted or standard biopsy for prostate-cancer diagnosis. $N$ Engl $J$ Med 2018;378:1767-77.

4. Loeb S, Vellekoop A, Ahmed HU, Catto J, Emberton M, Nam R, et al. Systematic review of complications of prostate biopsy. Eur Urol 2013;64:876-92.

5. McCormack $\mathrm{M}$, Duclos A, Latour M, McCormack $\mathrm{MH}$, Liberman D, Djahangirian $\mathrm{O}$, et al. Effect of needle size on cancer detection, pain, bleeding and infection in TRUS-guided prostate biopsies: A prospective trial. Can Urol Assoc J 2012;6:97-101.

6. Ukimura O, Coleman JA, de la Taille A, Emberton M, Epstein JI, Freedland SJ, et al. Contemporary role of systematic prostate biopsies: Indications, techniques, and implications for patient care. Eur Urol 2013;63:214-30.

7. Baco E, Rud E, Eri LM, Moen G, Vlatkovic L, Svindland A, et al. A randomized controlled trial to assess and compare the outcomes of two-core prostate biopsy guided by fused magnetic resonance and transrectal ultrasound images and traditional 12-core systematic biopsy. Eur Urol 2016;69:149-56.

8. Tyson MD, Arora SS, Scarpato KR, Barocas D. Magnetic resonance-ultrasound fusion prostate biopsy in the diagnosis of prostate cancer. Urol Oncol 2016;34:326-32.

9. Bjurlin MA, Mendhiratta N, Wysock JS, Taneja SS. Multiparametric MRI and targeted prostate biopsy: Improvements in cancer detection, localization, and risk assessment. Cent European J Urol 2016;69:9-18.

10. Ukimura O, Gross ME, de Castro Abreu AL, Azhar RA, Matsugasumi $\mathrm{T}$, Ushijima $\mathrm{S}$, et al. A novel technique using three-dimensionally documented biopsy mapping allows precise re-visiting of prostate cancer foci with serial surveillance of cell cycle progression gene panel. Prostate 2015;75:863-71.

11. Ukimura O, Desai MM, Palmer S, Valencerina S, Gross M, Abreu AL, et al. 3-dimensional elastic registration system of prostate biopsy location by real-time 3-dimensional transrectal ultrasound guidance with magnetic resonance/transrectal ultrasound image fusion. J Urol 2012;187:1080-6.

12. Cornud F, Roumiguié M, Barry de Longchamps N, Ploussard G, Bruguière $\mathrm{E}$, Portalez $\mathrm{D}$, et al. Precision matters in $\mathrm{MR}$ imaging-targeted prostate biopsies: Evidence from a prospective study of cognitive and elastic fusion registration transrectal biopsies. Radiology 2018;287:534-42.

13. Koelis; 2018. Available from: https://www.youtube.com/ watch? $\mathrm{v}=$ JolV6cURwLk. [Last accessed on 2019 Nov 02].
14. Ahmed HU, El-Shater Bosaily A, Brown LC, Gabe R, Kaplan R, Parmar MK, et al. Diagnostic accuracy of multi-parametric MRI and TRUS biopsy in prostate cancer (PROMIS): A paired validating confirmatory study. Lancet 2017;389:815-22.

15. Hansen NL, Barrett T, Kesch C, Pepdjonovic L, Bonekamp D, O'Sullivan R, et al. Multicentre evaluation of magnetic resonance imaging supported transperineal prostate biopsy in biopsy-naïve men with suspicion of prostate cancer. BJU Int 2018;122:40-9.

16. Panebianco V, Barchetti F, Sciarra A, Ciardi A, Indino EL, Papalia R, et al. Multiparametric magnetic resonance imaging vs. standard care in men being evaluated for prostate cancer: A randomized study. Urol Oncol 2015;33:17.e1-000000.

17. Hoeks CM, Somford DM, van Oort IM, Vergunst H, Oddens JR, Smits GA, et al. Value of 3-T multiparametric magnetic resonance imaging and magnetic resonance-guided biopsy for early risk restratification in active surveillance of low-risk prostate cancer: A prospective multicenter cohort study. Invest Radiol 2014;49:165-72.

18. Numao N, Yoshida S, Komai Y, Ishii C, Kagawa M, Kijima T, et al. Usefulness of pre-biopsy multiparametric magnetic resonance imaging and clinical variables to reduce initial prostate biopsy in men with suspected clinically localized prostate cancer. J Urol 2013;190:502-8.

19. de Gorski A, Rouprêt M, Peyronnet B, Le Cossec C, Granger B, Comperat E, et al. Accuracy of magnetic resonance imaging/ ultrasound fusion targeted biopsies to diagnose clinically significant prostate cancer in enlarged compared to smaller prostates. J Urol 2015;194:669-73.

20. Siddiqui MM, Rais-Bahrami S, Turkbey B, George AK, Rothwax J, Shakir N, et al. Comparison of MR/ultrasound fusion-guided biopsy with ultrasound-guided biopsy for the diagnosis of prostate cancer. JAMA 2015;313:390-7.

21. Valerio $M$, Donaldson I, Emberton $M$, Ehdaie B, Hadaschik BA, Marks LS, et al. Detection of clinically significant prostate cancer using magnetic resonance imaging-ultrasound fusion targeted biopsy: A systematic review. Eur Urol 2015;68:8-19.

22. Diamand R, Oderda M, Al Hajj Obeid W, Albisinni S, Van Velthoven R, Fasolis G, et al. A multicentric study on accurate grading of prostate cancer with systematic and MRI/US fusion targeted biopsies: Comparison with final histopathology after radical prostatectomy. World J Urol 2019;37:2109-17.

23. Delongchamps NB, Peyromaure M, Schull A, Beuvon F, Bouazza N, Flam $\mathrm{T}$, et al. Prebiopsy magnetic resonance imaging and prostate cancer detection: Comparison of random and targeted biopsies. J Urol 2013;189:493-9.

24. Hossack T, Patel MI, Huo A, Brenner P, Yuen C, Spernat D, et al. Location and pathological characteristics of cancers in radical prostatectomy specimens identified by transperineal biopsy compared to transrectal biopsy. J Urol 2012;188:781-5.

25. Xiang J, Yan H, Li J, Wang X, Chen H, Zheng X. Transperineal versus transrectal prostate biopsy in the diagnosis of prostate cancer: A systematic review and meta-analysis. World J Surg Oncol 2019;17:31.

26. de Gorski A, Rouprêt M, Peyronnet B, Le Cossec C, Granger B, Comperat E, et al. Accuracy of magnetic resonance imaging/ ultrasound fusion targeted biopsies to diagnose clinically significant prostate cancer in enlarged compared to smaller prostates. J Urol 2015;194:669-73.

27. $11^{\text {th }}$ International Symposium on Focal Therapy and Imaging in Prostate and Kidney Cancer; 2019. Available from: https://www. focaltherapy.org/2019/NEWSLETTERS/vol1-sept18.html. [Last accessed 2020 Jan 7]. 